\title{
D'imagier à ymaginero : la présence de sculpteurs septentrionaux en Espagne aux $\mathrm{XV}^{\mathrm{e}}-\mathrm{XVI}^{\mathrm{e}}$ siècles
}

\author{
María Teresa Rodríguez Bote
}

\section{(2) OpenEdition \\ Journals}

Édition électronique

URL : https://journals.openedition.org/cem/14535

DOI : 10.4000/cem. 14535

ISSN : 1954-3093

Éditeur

Centre d'études médiévales Saint-Germain d'Auxerre

\section{Référence électronique}

María Teresa Rodríguez Bote, « D'imagier à ymaginero : la présence de sculpteurs septentrionaux en Espagne aux xve-xvie siècles », Bulletin du centre d'études médiévales d'Auxerre | BUCEMA [En ligne], 20.2 | 2016, mis en ligne le 06 mars 2017, consulté le 22 septembre 2022. URL : http:// journals.openedition.org/cem/14535; DOI : https://doi.org/10.4000/cem.14535

Ce document a été généré automatiquement le 22 septembre 2022.

\section{(c) (†) (ㅇ)}

Creative Commons - Attribution - Pas d'Utilisation Commerciale - Partage dans les Mêmes Conditions 4.0 International - CC BY-NC-SA 4.0

https://creativecommons.org/licenses/by-nc-sa/4.0/ 


\title{
D'imagier à ymaginero : la présence de sculpteurs septentrionaux en Espagne aux $\mathrm{XV}^{\mathrm{e}}-\mathrm{XVI}^{\mathrm{e}}$ siècles
}

\author{
María Teresa Rodríguez Bote
}

1 La "géographie de l'art » est presque une nouvelle discipline dans les études et recherches en histoire de l'art, elle a pris son essor ces dernières années ; beaucoup de rencontres scientifiques et de publications sur le sujet en témoignent ${ }^{1}$. Quelle est la raison de ce nouvel intérêt? Peut-être les événements actuels et la mondialisation ont poussé la communauté scientifique à réfléchir sur le phénomène de la mobilité et sur la communication artistique pendant des siècles. En outre, en approfondissant le sujet, nous

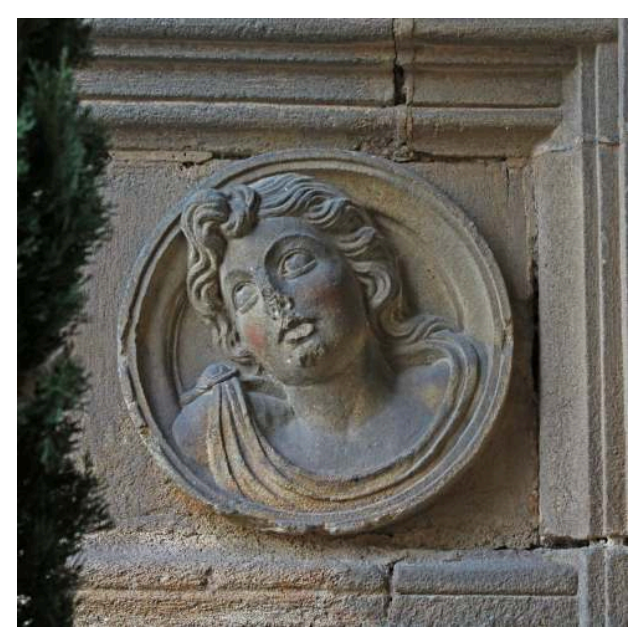
observons que le concept d'internationalisation n'est pas si actuel qu'on pourrait l'imaginer a priori ; et que cette idée d'un art ou d'un style «national » devient dépassée. En témoignent les débats sur la précision et les nuances sémantiques des termes tels que "limite ", "frontière », « transfert », « échange » et « influence ».

2 Le flux migratoire des Européens qui se sont déplacés vers les royaumes hispaniques pendant le $\mathrm{XVI}^{\mathrm{e}}$ siècle est fort connu. L'historiographie a en effet étudié ce phénomène à travers de nombreuses recherches ${ }^{2}$. Il s'agit d'un courant migratoire qui commence au Moyen Âge, qui croît à partir de la seconde partie du $\mathrm{xv}^{\mathrm{e}}$ siècle et perdure pendant tout l'Ancien Régime. Étant donné le nombre croissant des artistes étrangers - nous en avons repéré plus de deux cents concentrés seulement en Castille - l'intérêt que le sujet représente est spécialement remarquable ${ }^{3}$. Parmi ces artistes, on compte des 
représentants de toutes les disciplines et provenances, mais, surtout, au moins dans le domaine de la sculpture, ce sont les Français les plus nombreux. Traditionnellement, les maitres italiens sont, tout au long des temps modernes, ceux qui attirent l'attention des chercheurs ; on constate cependant que ce rôle est plus partagé chez les artistes.

Quoi qu'il en soit, leur contribution à l'art hispanique de cette époque est incontestable et témoigne de l'énorme mobilité des artistes. En même temps, ceci nous pose des questions concernant les liens potentiels entre les divers foyers et traditions artistiques: peut-on parler des influences réciproques? Tandis que ces artistes exogènes importent des nouveautés esthétiques et des techniques considérables, s'adaptent-ils au système et aux préférences de la société et clientèle castillanes? S'agit-il d'une dialectique entre langages différents ou d'une fusion ? À quel point sontils des acteurs qui renouvellent les tendances du moment? Quelles raisons poussent les commanditaires à les choisir? Ce qui est clair c'est que ces déplacements constituent sans aucun doute un pas en avant dans les démarches d'une identité artistique pas si nationale, plutôt européenne, et qui, en plus, montrent l'évolution du concept de « frontière » pendant l'Ancien Régime.

\section{Le flux migratoire des huchiers pendant le $x^{e}$ siècle}

Presque une vingtaine de tailleurs ou, pour être plus précise, des « huchiers », comme ils étaient appelés à l'époque, passent les Pyrénées et annoncent le devenir du siècle suivant. $\mathrm{Au} \mathrm{Xv}^{\mathrm{e}}$ siècle, ils ont appartenu en particulier à quelques-uns des centres artistiques les plus importants du moment: Pampelune en Navarre et, en Castille, Tolède et Burgos. Dans ces ateliers, ils ont travaillé à la décoration des cathédrales.

5 Le chantier de Pampelune, en particulier, a fortement attiré les artistes et donc un atelier se crée où les artistes exogènes jouent un rôle considérable ${ }^{4}$. À cet égard, il convient de rappeler le fort lien politique et diplomatique que l'ancien Royaume de Navarre entretient depuis le Moyen Âge avec la Bourgogne et la couronne française, de même que la proximité des Pyrénées et les transferts culturels continus.

6 Ces «huchiers » maîtrisaient très probablement la sculpture sur bois, mais aussi sur pierre $^{5}$; ceux qui travaillent en Castille n'apparaissent pas seulement comme des " entalladores", mais aussi comme des « canteros ». Ces mêmes huchiers gagnent plus tard une excellente réputation. Ce prestige est dû aux œuvres qui nous sont restées, mais aussi à l'historiographie ; on peut citer comme exemples Pedro et Juan Guas - père et fils originaires de Bretagne -, Juan de Cologne, Egas Cueman ou Gil Siloe, qui figurent parmi les plus célèbres. Il faut également ajouter d'autres tailleurs, qui, bien qu'ils ne soient pas directement reliés à un foyer en particulier, restent méritants de valeur et d'intérêt artistique.

7 Ainsi Michel Lochner ${ }^{6}$, un de ces rares sculpteurs venus depuis l'autre côté des Pyrénées, exerce son métier en Catalogne pendant cette période. S'il y a si peu de tailleurs en Catalogne, c'est pour des raisons multiples : d'une part, ce n'est pas si loin d'Avignon, d'Arles, d'Aix ou encore de Nîmes, qui constituaient des villes avec de gros chantiers et des ateliers où travailler; tandis que dans cette zone de la Péninsule les entreprises artistiques n'étaient pas si nombreuses et coûteuses en comparaison avec les Castillanes. En plus, il y a aussi de possibles allers-retours à travers la Jonquère, en d'autres mots, les artistes qui étaient descendus pendant quelque temps, retournaient 
en France sans laisser aucune trace documentaire. Cette pratique nomade était assez courante à l'époque: Hannequin de Bruxelles, le frère d'Egas Cueman, s'installe en premier lieu en Aragon, puis il voyage à Tolède et finalement reste à Léon. De la même façon, au sud, l'œuvre de Lorenzo Mercadante de Bretagne peut être repérée dans divers endroits du sud-ouest, à Aracena, Fregenal de la Sierra et Séville ${ }^{7}$.

En tout état de cause, ils peuvent tous être inclus dans le courant hispano-flamand, étant donné qu'ils satisfont les critères stylistiques de la sculpture gothique tardive en vogue dans les territoires d'accueil. À cette époque, ils n'apportent aucune nouveauté esthétique, mais plutôt iconographique: par exemple, le maître Rodrigo Alemán s'inspire des proverbes assez populaires à l'époque dans les régions septentrionales de l'Europe ${ }^{8}$ (fig. 1).

Fig. 1 - Stalles de la cathédrale de Ciudad Rodrigo, par Rodrigo Alemán (avant 1503)

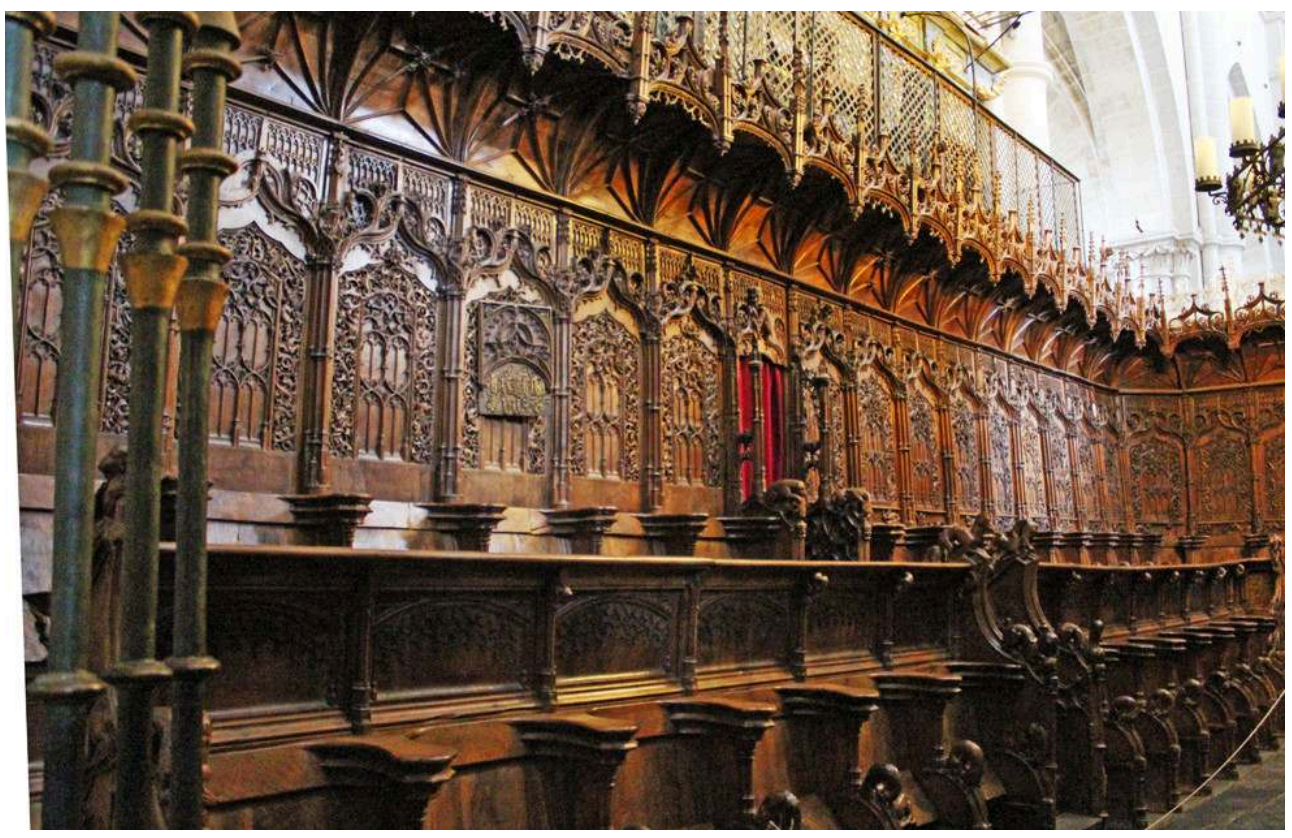

C'est l'excellente réputation que les ateliers du Nord avaient déjà qui fait qu'on les engage. En revanche, certains auteurs ont montré l'importation de techniques peu communes dans la sculpture ibérique9.

\section{Quelques difficultés concernant les tailleurs du $\mathrm{XVI}{ }^{\mathrm{e}}$ siècle}

Parmi tous ces artistes qui arrivent au long du $\mathrm{XVI}^{\mathrm{e}}$ siècle on trouve un groupe assez nombreux de tailleurs provenant du nord de la France actuelle et, en particulier, pendant les décennies centrales du siècle. On y trouve aussi des ferronniers (rejeros), des vitriers, des peintres et toute sorte d'autres métiers. Pour le moment, nous ne parlerons que des tailleurs, qui sont le collectif le plus nombreux. Nous avons repéré des références documentaires de plus de trois cents d'entre eux en activité dans les royaumes de Castille et d'Aragon. Afin de définir ce groupe, nous tiendrons compte exclusivement de ceux qui sont nés et qui ont appris leur métier hors d'Espagne ; la génération suivante, même si elle a reçu une formation dans le foyer familial, a été 
beaucoup plus exposée aux vagues stylistiques autochtones hispaniques. Logiquement, nous excluons tous les artistes, qui, ne travaillant que pour des commanditaires espagnols, particulièrement pour la Couronne, importent leurs œuvres de l'au-delà des frontières et n'ont jamais vécu sur le territoire espagnol ; c'est le cas, par exemple, d'Adrián de Vries.

11 En affinant le nombre de tailleurs dont nous parlons, nous faisons face à certaines difficultés, telles que, premièrement, la dénomination de leur activité. En espagnol, il y a de très nombreux termes assez spécifiques pour nommer chacune des fonctions dans un atelier, où chaque ouvrier a d'ordinaire la même occupation. Pourtant, parfois, afin de mieux organiser le travail et le temps, ces collègues se sous-traitent ou partagent les commandes et projets entre eux; cette pratique n'est pas toujours très bien vue chez les commanditaires ${ }^{10}$. En outre, un seul ouvrier ou un maître peut habituellement exercer des fonctions différentes.

Deuxièmement, en dehors d'un anonyme procédant de Saint-Omer (Pas-de-Calais) travaillant à Llanes (Asturies), la plupart des identifiés peuvent poser des problèmes, étant donné les nombreuses variantes graphiques pour le nom d'une même personne. D'ailleurs, les écrivains publics enregistrent les prénoms à partir de l'oral ou, de façon plus commune encore, les hispanisent. Ces sculpteurs signent aussi en remplaçant leur nom par celui de leur ville, de leur pays d'origine, etc. Par exemple, Jan Yneres, le maitre tailleur des stalles de la cathédrale de Zamora (fig. 2), est connu comme Juan de Bruselas, même si dans certains registres il signe avec son nom original ${ }^{11}$.

Fig. 2 - Stalles de la cathédrale de Zamora, par Juan de Bruselas (1502)



13 Pour la plupart d'entre eux, on repère assez facilement qu'il s'agît d'un même artiste avec des noms semblables; mais dans d'autres cas, établir un lien sans avoir d'information suffisante ou plus précise reste relativement risqué. L'un des cas le plus curieux, selon nous, est le frère Juan de Beauvais, parce que le toponyme apparaît écrit de toutes les formes imaginables (Beauvais, Beauves, Beures...) ; cependant il était - et 
reste encore aujourd'hui - plus connu comme « el fraile » ou « fray Juan ». Un autre nom, qui admet jusqu'à huit variantes écrites, est Vigarny ${ }^{12}$.

De la même façon, il y a différents artistes dont les noms et prénoms sont identiques et qui travaillent à différents endroits ; comment profiler ces personnalités ? Vigarny luimême peut être confondu avec un autre Felipe de Bourgogne, actif à Oñate et à Logroño. D'autre part, on trouve deux Cornielis de Hollande, le premier habite à Burgos, le second en Galice. Lorsque les données documentaires n'apportent pas d'informations suffisantes, seule une profonde analyse technique et stylistique pourrait nous donner une solution.

Un dernier paramètre à surmonter est le manque de renseignements. Certes, certains d'entre eux ont été des artistes remarquables et ont donc attiré l'attention tout au long de l'historiographie. Nous retrouvons parmi eux les cas de Jean de Joigny ou Étienne Jamet. Néanmoins, nombreux sont ceux, qui, malgré la qualité de leur travail, ne sont guère connus; on n'en trouve que quelques mentions indirectes et isolées. C'est pour cela que nous ne rejetons pas du tout l'hypothèse d'en trouver encore d'autres. À ceci, s'ajoutent tous ceux qui restent trop peu de temps sur la Péninsule ou qui font des allers-retours et qui, par conséquent, ne laissent aucune trace.

\section{Le collectif des tailleurs}

On peut nettement définir des caractères que presque tous ces sculpteurs partagent grosso modo. La plupart des tailleurs sont originaires des ateliers de certaines régions les plus septentrionales : la Normandie, la Picardie, la Champagne, la Bourgogne, la Loire, l'île-de-France, la Lorraine, le Pays basque, voire les Flandres et l'Allemagne.

Du côté méditerranéen, nous ne pouvons pour le moment confirmer la présence que de trois personnes : Francisco de Marseille, Guillén de Carcassonne et Juan d'Avignon en Navarre ${ }^{13}$. Selon nous, certaines raisons peuvent expliquer ce phénomène : la puissance économique et le marché artistique dans ces foyers du Midi pourraient offrir plus d'opportunités et de stabilité aux artistes autochtones (et exogènes aussi); ils n'auraient par conséquent pas une trop forte pression pour s'en aller. Il est aussi possible que les méridionaux aient préféré déménager dans les républiques italiennes, en raison des affinités culturelles, de l'intérêt archéologique, de la mode artistique du moment, etc. On a récemment vérifié les chiffres sur l'immigration arrivée dans les villes d'Avignon, d'Arles et d'Aix-en-Provence pendant le $\mathrm{xv}^{\mathrm{e}}$ siècle; plus précisément ceux relatifs aux bâtisseurs venus du Massif central, qui peuvent être appliqués aux tailleurs et démontrent en plus le pouvoir d'attraction des entreprises provençales et du Languedoc-Roussillon ${ }^{14}$. Un autre foyer remarquable est celui de Toulouse, qui constitue un fort pôle d'attraction ${ }^{15}$. Cette ville est aussi un des principaux arrêts lors du déplacement des artistes vers le sud, ce qui a des conséquences artistiques.

En revenant sur le reste, on peut ajouter que tous ont une formation italianisante. Ils partent du nord sous prétexte de se rendre à Compostelle, comme Étienne Jamet ${ }^{16}$; mais, une fois passés les Pyrénées, ils se répandent dans toute la Péninsule. Il y en a même un, Louis Van der Vule, qui voyage des Pays-Bas jusqu'aux îles Canaries ${ }^{17}$. En effet, à peu près une centaine - plus ou moins la moitié des arrivés - travaille dans des ateliers du quart nord-est - Saragosse, Tarazona, Navarre, Pays basque et Rioja -, où ils trouvent une clientèle assez prospère qui leur garantit des commandes continues, 
tandis qu'ils contribuent à réactiver une densité de population assez basse ${ }^{18}$. À ce propos, on peut citer les frères Beaugrant ${ }^{19}$ et les Imbert: ils fondent des ateliers familiaux, où non seulement leurs enfants mais aussi des apprentis autochtones reçoivent une formation renaissante ; ce qui a enrichi le paysage artistique et a permis par ailleurs de répandre de nouveaux modèles plastiques.

19 L'autre moitié s'installe dans les villes principales de Castille et d'Andalousie; certains mènent une vie nomade en participant à divers chantiers dans tout le pays ou joignent des ateliers semi-itinérants. Ceux qui partent en Amérique après un séjour en Espagne méritent une étude à part. Jusqu'à présent, nous n'avons repéré que deux peintres et un architecte ayant un tel parcours.

Revenons à ce qui nous concerne. À notre avis, les raisons qui les poussent à traverser les Pyrénées sont essentiellement pécuniaires: ils ont souffert de la faim et de l'incertitude à cause des conflits religieux et politiques issus de la Réforme. C'est pourquoi ils se décident à partir, sans doute encouragés par les nouvelles reçues d'autres travailleurs qui pouvaient avoir réussi d'ailleurs antérieurement. Les richesses des ports et des foires espagnoles, outre la croissante demande d'artistes, deviennent un appel et une opportunité pour améliorer leurs conditions de vie. Il faut également dire, que, sur les territoires hispaniques, la concurrence n'était pas si forte, étant donné qu'il y avait là moins de sculpteurs et plus de commandes; sans compter que les salaires étaient plus élevés en Castille ${ }^{20}$.

21 Alors que dans les ateliers septentrionaux, l'organisation des corporations et le nombre de tailleurs et d'imagiers entraînent une certaine pression et rigidité; en Espagne, le nombre des sculpteurs est assez faible et, par conséquent, le métier n'est pas si corseté. D'ailleurs, surtout en Castille, on trouve aussi plus de commanditaires avec de plus gros budgets. Même si ces sculpteurs exogènes doivent faire face aux autochtones, cet effort vaut la peine et même cela constitue sans doute, pour certains, une espèce de libération.

\section{Systèmes d'accueil}

22 Le collectif des travailleurs qui se déplacent au $\mathrm{xvI}^{\mathrm{e}}$ siècle et, en particulier, les sculpteurs, a réussi à s'intégrer. La clientèle pour laquelle ils travaillent est sans doute un bon exemple : des laïcs, mais aussi les membres du clergé ou des institutions ecclésiastiques, le démontrent. Il n'y avait aucun inconvénient à leur faire une commande avec un contenu religieux ou dans un but liturgique, étant donné que l'artiste a un bon savoir-faire et que l'exécution du travail s'adapte aux exigences et conditions convenues. Jean de Joigny a reçu de grands éloges au fil de sa carrière ; à l'instar de Vigarny, bien considéré par Diego de Sagredo et appelé «mon frère maître Philippe» par son beau-frère, Alonso de Covarrubias, un des plus remarquables architectes à l'époque ${ }^{21}$.

Dans leurs vies personnelles, ils se lient tous d'amitié là où ils habitent; au sein des ateliers, même si normalement ils travaillent avec quelqu'un du même pays, ils collaborent et forment des apprentis espagnols. Ils ne restent parfois sur la Péninsule que pendant une saison, après quoi ils rentrent chez eux ; mais, d'habitude, ils créent un réseau de contacts endogamique et gardent des liens avec leurs villes d'origine. À ce propos, on peut citer comme exemple Nicolás de Hollande, qui se marie avec María Enríquez Bolduque en 1565 - fille de Mateo de Bolduque (Bois-le-Duc), qui avait établi 
un clan familial. Par ailleurs, vingt ans avant, lors du baptême de l'aîné de la famille, Pedro, c'est Jerónimo del Corral qui est le témoin ${ }^{22}$.

De surcroît, la quantité d'accords professionnels parmi eux démontre d'ailleurs ces liens. C'est ainsi qu'aux côtés de Vigarny, c'est León Picard qui est habituellement en charge de la peinture ${ }^{23}$; frère Juan de Beauvais, déjà mentionné auparavant, acquit sa formation chez Gabriel Joly et travaille plus tard avec Pierres Picart, qui, en outre, vient de l'atelier de Vigarny ${ }^{24}$. Il est assez commun de trouver des compatriotes travaillant ensemble, c'est le cas pour la façade de l'université d'Alcalá de Henares ${ }^{25}$, mais aussi des stalles du monastère de Saint-Marc à León ${ }^{26}$ et de l'hôtel de ville à Séville ${ }^{27}$.

En revanche, nombreux sont ceux qui se marient avec des Espagnoles et s'installent dans les domaines de la monarchie hispanique. Ce genre d'union leur apporte stabilité et sécurité juridique, particulièrement concernant deux aspects : tout d'abord, presque tous les arrivés en Espagne sont jeunes et célibataires, avec l'intention de s'établir; d'une autre part - et peut-être la plus importante - à partir de l'époque du roi Ferdinand, des ordres d'expulsion sont promulgués contre les Français, malgré la difficulté à exécuter ces décrets, ainsi que des exemptions dont bénéficient beaucoup d'entre eux. Ce n'est qu'au XVII siècle que la monarchie, soit à cause de la peur des réformistes, soit pour des raisons de protectionnisme économique, commence à mettre en œuvre des mesures plus draconiennes, telles que des interdictions, des expulsions (effectives), des embargos ou confiscation des biens, etc. ${ }^{28}$.

\section{Conséquences pour l'histoire de l'art}

On pourrait, en principe, considérer que la formation de ces tailleurs appartient au gothique tardif, qui suit l'exemple de Claus Sluter ou des huchiers traditionnels et des imagiers du Nord. Cependant, même si cette esthétique leur semble assez proche, ils ont déjà bien appris et importent en effet des traits fortement italiens. Ils offrent, à vrai dire, une solution entre les deux tendances de la sculpture du moment, l'Italienne et la Flamande ; et ce sens de la mesure et de l'équilibre devient vraisemblablement la clé de l'acceptation chez leur nouvelle clientèle. La conséquence de cette médiation est une sculpture belle et harmonieuse, un "doux naturalisme, stylisé avec légèreté29 ", en d'autres mots, une synthèse de deux traditions où le meilleur de chacune est exploité. Dans l'Espagne du $\mathrm{xVI}^{\mathrm{e}}$ siècle, le goût flamand était bien répandu et les formes italiennes étaient par conséquent difficiles à introduire; en revanche, l'assimilation d'un italianisme plus tempéré était plus simple. À ceci, s'ajoute l'évolution au cours du temps, lorsqu'on observe une certaine tendance à se rapprocher de la sculpture hispanique, soit par influence d'autres artistes autochtones, soit afin de s'assurer plus de travail et de commandes.

À vrai dire, tous ces maîtres travaillent coude à coude avec des tailleurs (officiers ou apprentis) et des ateliers espagnols, de telle manière qu'ils forment de petits foyers «afrancesados » là où ils s'installent. Il $\mathrm{y}$ a de nombreux sculpteurs espagnols dont l'œuvre adopte un air septentrional, mais pour le moment nous ne ferons qu'en mentionner quelques-uns des plus représentatifs et clairs: les Corral de Villalpando, qui emploient des motifs décoratifs d'origine gauloise, tels que les effets picturaux des bas-reliefs, les micro-architectures en fuite et suspendues et les supports anthropomorphes ${ }^{30}$. L'imagier Pedro de Paz s'imprègne d'ailleurs des recours nordiques lorsqu'il s'inspire des modèles du retable des Bolduque à Cáceres ${ }^{31}$. 
La même chose se passe avec d'autres typologies iconographiques qu'ils contribuent à répandre, en particulier la Mise au tombeau, mais aussi les Lamentations sur le corps du Christ - déjà introduites dans les royaumes ibériques à la fin du Moyen Âge - et qui avaient une grande diffusion au-delà des Pyrénées ${ }^{32}$, où l'on trouve de multiples exemples remarquables par leur beauté extraordinaire et leur raffinement plastique. Nous n'avons pas pour objectif d'établir un parallèle direct entre ces œuvres, que nous mentionnons en guise d'exemples représentatifs ; bien au contraire, nous soulignons la quantité notable de Mises au tombeau en Espagne issues d'ateliers d'origine gauloise. Au-delà de l'ensemble sculptural de Jean de Joigny, on trouve également deux petits reliefs d'une grande valeur artistique : le premier, taillé par Pierres Picart (fig. 3) et, le second, par frère Rodrigo de Hollande (fig. 4), destinés à des retables castillans.

Fig. 3 - Mise au tombeau. Relief, par Pierres Picart, vers 1560

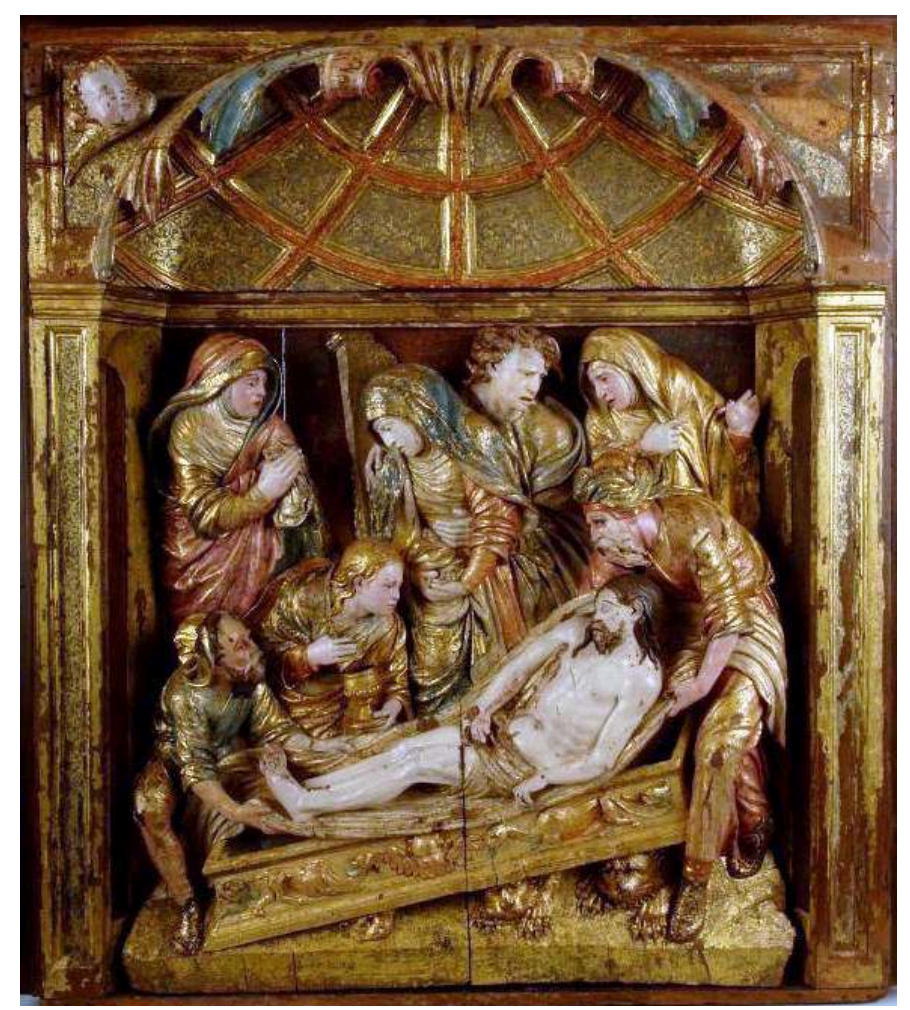

Museo Nacional de Escultura, Valladolid 
Fig. 4 - Mise au tombeau. Relief, par Rodrigo de Holanda, vers 1530

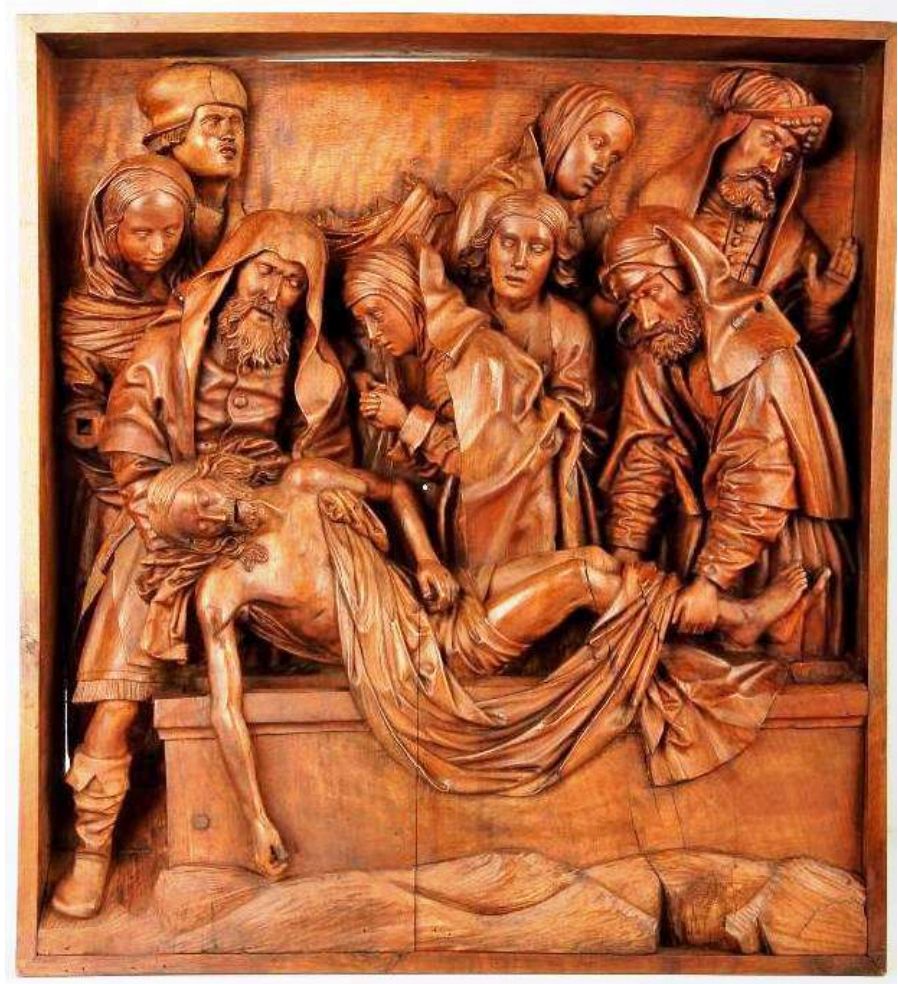

Museo Nacional de Escultura, Valladolid

Par ailleurs, le médaillon est un autre élément décoratif dont la propagation est une contribution de ce collectif. On peut le trouver dans la décoration monumentale des façades, des retables, des stalles et du mobilier liturgique en général. D'origine italienne, le médaillon est issu de l'intérêt pour le collectionnisme numismatique renaissant et pour les imagines clipeatae de l'Antiquité ; il s'est fortement répandu dans les chantiers français du xvI e siècle, comme le château de Gaillon, ou dans la décoration toulousaine ${ }^{33}$. Même si les premiers médaillons en Espagne sont ceux à La Calahorra (1509-1512) $)^{34}$, la formation de tailleurs a sûrement dû s'imprégner de cette culture visuelle et donc se reflète dans les projets péninsulaires auxquels ils ont participé au cours de leurs vies. Le médaillon est assez répandu dans l'architecture du $\mathrm{XvI}^{\mathrm{e}}$ siècle ; certains des tailleurs français - mais aussi quelques tailleurs espagnols -, qui travaillent à Alcalá de Henares, Séville et Salamanque, sont aussi repérés aux chantiers de Plasencia ${ }^{35}$, d'où proviennent les exemples de la Puerta Sacra, par Guillén, Jacques et autres tailleurs (1522-1558) (fig. 5 et 6). 
Fig. 5 - Détail (médaillon) de la Puerta Sacra, par Guillén, Jacques et autres tailleurs (1522-1558)



Cathédrale de Plasencia

Fig. 6 - Détail (médaillon) de la Puerta Sacra, par Guillén, Jacques et autres tailleurs (1522-1558)

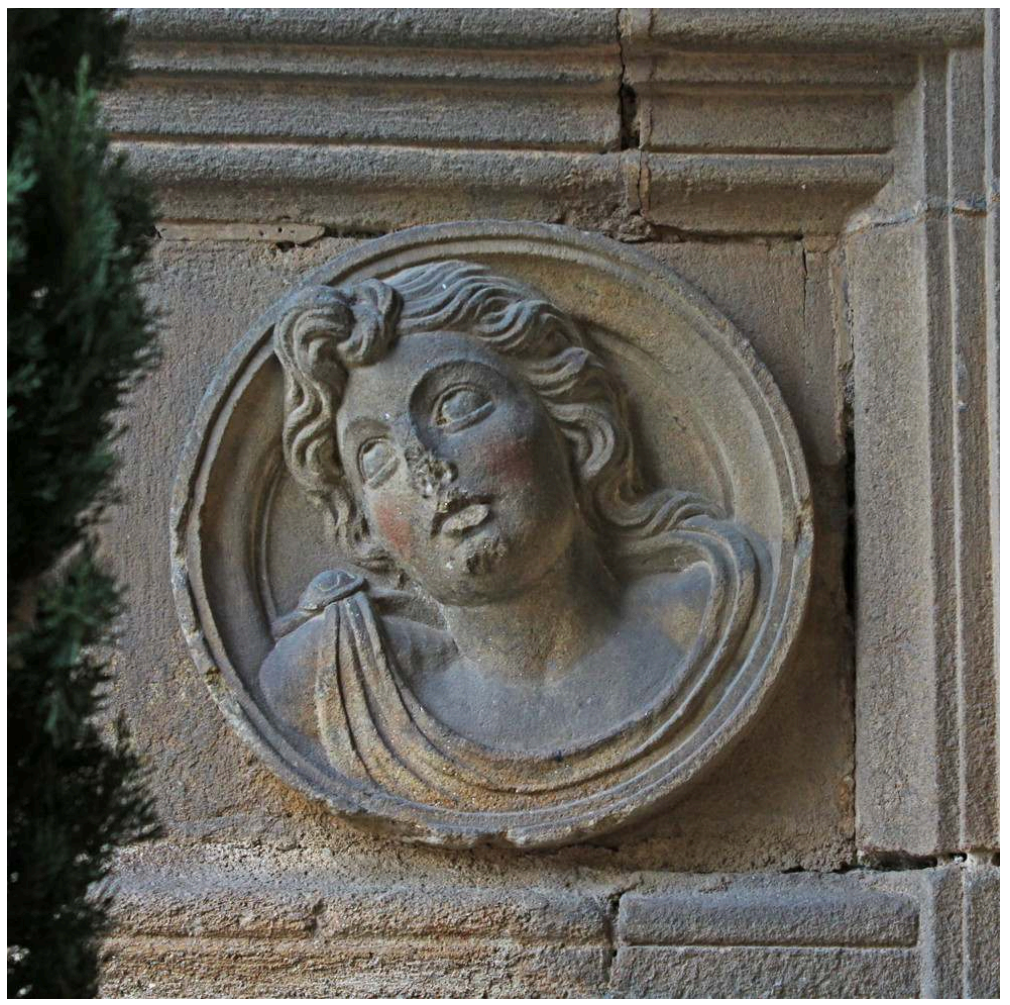

Cathédrale de Plasencia 
Il semble assez complexe - voire impossible, pourrions-nous affirmer - de reconnaître l'apport de chacun des artistes de manière individuelle, car au sein d'un même atelier ils travaillent en équipe. Néanmoins, ceci n'empêche pas d'attester cette influence nordique dans la sculpture hispanique de la Renaissance. À ceci s'ajoute, en dernier lieu, le rôle de ces tailleurs quant à l'emploi de nouveaux modèles de composition et à la diffusion de gravures procédant des imprimeurs septentrionaux. C'est pourquoi nous pouvons sans aucun doute prendre en considération une Renaissance à la française dans les royaumes de Castille et d'Aragón, comme l'historiographie la plus récente l'a établi36.

\section{Conclusions}

En bref, le courant sculptural nordique dans l'Espagne du $\mathrm{xVI}^{\mathrm{e}}$ siècle doit être tenu comme un grand flux migratoire, composé de tailleurs, la plupart d'entre eux provenant du nord de la France, afin de développer leur profession et attirés par la croissance économique de la monarchie hispanique de l'époque. On peut parfois trouver des difficultés à établir le profil de leur activité et leur identité, puisqu'ils signent d'habitude de différentes formes : avec leur ville ou région d'origine, mais aussi avec le prénom en français originel, etc. La plupart peuvent toutefois être identifiées aisément.

Même si cette tendance a démarré bien avant, au Moyen Âge, c'est néanmoins à partir de la seconde moitié $d u x^{e}$ siècle que ce mouvement démographique augmente et perdure pendant tout l'Ancien Régime, mais spécialement dans le tiers central du $\mathrm{xVI}^{\mathrm{e}}$ siècle. Ils ont déjà acquis une formation dans ateliers dont ils sont issus, mais ceci ne les empêche pas de s'adapter parfaitement aux exigences stylistiques du marché. Ils contribuent, en effet, à répandre le nouveau langage plastique des formes italiennes en Espagne.

Mis à part leur rôle dans l'introduction des motifs renaissants de la décoration monumentale, comme le médaillon, ils enrichissent aussi le paysage artistique à travers l'établissement d'ateliers en Espagne, où des apprentis autochtones reçoivent une formation au fil des nouveautés "à l'antique». Quelques-uns d'entre eux sont des artistes remarquables et profitent d'une très bonne réputation professionnelle ; c'est le cas d'Étienne Jamet, de Jean de Joigny, de Pierres Picart, des Bolduque, etc. Cependant, nombre d'entre eux, malgré la qualité de leur travail, ne sont guère connus aujourd'hui; une recherche plus profonde sur le sujet devient, par conséquent, nécessaire.

Reçu : 5 août 2016 - Accepté : 9 décembre 2016

Bulletin du centre d'études médiévales d'Auxerre | BUCEMA, 20.2 | 2016 


\section{NOTES}

1. Cette recherche est réalisée dans le cadre du «Programa propio III de la Universidad de Salamanca - Contratos predoctorales». Quelques-unes des études les plus importantes sur les migrations au cours de l'histoire sont les suivantes: T. W. GAEHTGENS, «Discours d'ouverture ", Berlin, 1992 ; B. JoYeux, "Les transferts culturels. Un discours de la méthode ", Hypothèses (2002-2003), p. 151-161 ; T. DACOSTA KAUFMANN, Toward a Geography of Art, Londres/Chicago, 2004 ; S. CIRIACONO, "Migration, Minorities, and Technology Transfer in Early Modern Europe ", The Journal of European Economic History, 34 (2005), p. 43-64 ; Global Art History and the Peripheries, Paris, 2013.

2. Voir les travaux remarquables sur l'immigration vers les royaumes hispaniques au cours de l'Ancien Régime: J. OLLERo BUtLeR, "Los flamencos en la España del siglo XVI ", Anuario del Departamento de Historia y Teoría del Arte, 2 (1990), p. 173-177; Mª B. VILLAR GARCíA et P. PEZZI CRISTÓBAL (dir.), Los extranjeros en la España moderna : actas del I Coloquio internacional, Málaga, 2003, en particulier l'article de J. A. SALAS AUSÉNS, «Leyes de inmigración y flujos migratorios en la España Moderna », p. 681-697 ; F. J. LORENZO PINAR, «La presencia de extranjeros en la ciudad de Salamanca en la primera mitad del siglo XVII » et «La presencia de extranjeros en Zamora en el siglo XVII », in J. A. BLANCO RODRíGUEZ (éd.), La emigración castellana y leonesa en el marco de las migraciones españolas, Zamora, 2011, p. 85-124 et 125-162 ; A. VIÑA BRITO, "Los flamencos en Canarias en el siglo XVI. ¿Una comunidad extranjera? Especificidades en la Isla de La Palma », Revista de historia canaria, 194 (avril 2012), p. 161-191 ; L. RUIZ MOLINA, J. J. RUIZ IBÁÑEZ et B. VINCENT (éd.), El Greco... y los otros. La contribución de los extranjeros a la monarquía hispana, 1500-1700, Murcie, 2015.

On peut également mentionner les études particulières sur l'immigration française : J. NADAL OLLER et E. GIRALT Y RAVENTÓs, La population catalane de 1553 à 1717. L'immigration française et les autres facteurs de son développement, Paris, 1961; A. POITRINEAU, « La inmigración francesa en el reino de Valencia », Moneda y Crédito, 137 (1976), p. 103-133 ; J. A. SALAS AUSÉNS, « La inmigración francesa a Aragón en la Edad Moderna », Estudios del Departamento de Historia Moderna (1985-1986), p. 51-77 ; Les Français en Espagne à l'époque moderne : XVI ${ }^{e}$-XVIII ${ }^{e}$ siècles, Paris, 1990 ; SALAS AUSÉNS, « Migraciones francesas en España (ss. XVI-XIX) », in D. L. GONZÁLEZ LOPO et A. EIRAS ROEL (coord.), La inmigración en España: actas del coloquio, Saint-Jacques-de-Compostelle, 2004, p. 77-102 ; P. J. MONTEANO SORBET, «Vascos y franceses en la Tudela de mediados del siglo XVI», Príncipe de Viana, 234 (2005), p. 111-133 ; M. BOEGLIN, « Luteranos franceses en la España de los Austrias. Aspectos culturales de un conflicto religioso ", in M. BRUÑa CUEVAS, $M^{a}$ de G. CABALLOS BEJANO, I. ILLANES ORTEGA, C. RAMírEZ GÓMEZ et A. RAVENTÓs BARANGÉ (coord.), La cultura del otro: español en Francia, francés en España, Séville, 2006.

3. Parmi les études les plus signifiantes portants exclusivement sur des artistes venus au-delà des Pyrénées, on trouve : S. ALCOLEA GIL, «Vitalidad artística del Camino de Santiago en el siglo XVI », Príncipe de Viana, 25, 96 (1964), p. 201-212 ; P. S. мАROTо, « Die Kunst der Krone - Flandern und Kastilien », in T. H. BORCHERT (éd.), Jan Van Eyck und seine Zeit. Flämische Meister und der Süden 1430-1530, 2002, p. 143-165 ; M. CABAÑAS BRAVo (éd.), El arte foráneo en España. Presencia e influencia, Madrid, 2005 ; J. LUGAND, Les échanges artistiques entre la France et l'Espagne (XVe-fin XIXe siècles), Perpignan, 2012 ; J. DUBOIS, J.-M. GUILLOUËT et B. VAN DEN BOSSCHE (éd.), Les transferts artistiques dans l'Europe gothique. Repenser la circulation des artistes, des cuvres, des thèmes et des savoir-faire (XII ${ }^{e}-\mathrm{XVI}^{e}$ siècle), Paris, 2014.

4. J. GoÑ gazTAmbide, « El coro de la catedral de Pamplona », Príncipe de Viana, 27, 104-105 (1966), p. 321-325. 
5. M. J. REDONDO CANTERA, «L'apport français à la sculpture de la Renaissance en Castille. Réflexions sur le style et les matériaux ", in La sculpture française du XVI ${ }^{e}$ siècle. Études et recherches, Marseille, 2011, p. 147 ; S. D. DAUSSY, «L'affirmation du métier de sculpteur à Amiens dans la première moitié du XVI $\mathrm{e}^{\mathrm{e}}$ siècle ", in La sculpture française du XVI e siècle..., ibid., p. 46-55.

6. J. MOLINA FIGUERAS, "Rutas artísticas y cultura visual en Cataluña », in F. CHECA et B. J. GARCíA GARCíA (éds.), El arte en la corte de los Reyes Católicos. Rutas artísticas a principios de la Edad Moderna, Madrid, 2005, p. 115-143.

7. On peut trouver plein de parutions récentes sur cet artiste; néanmoins le recueil le plus complet sur l'œuvre de Mercadante est celui de M. A. MARTín SÁNCHEZ, El imaginero Lorenzo Mercadante. Estudio de la obra y claves de su huella en la Virgen de las Nieves de la isla de Gran Canaria, Tenerife, 2009.

8. I. MATEO GÓMEZ, Temas profanos en la escultura gótica española. Las sillerías de coro, Madrid, 1979.

9. E. AGUAdo GUARDIOLA, A. M. MUÑOZ SANCHO et J. IBÁÑEZ FERNÁNDEZ, « Transferts des techniques de taille et de polychromie de la sculpture en pierre bourguignonne dans la péninsule Ibérique. Apports pour leur conservation, restauration et entretien ", in Les transferts artistiques..., op. cit., p. 95-97.

10. J. ARRANZ ARRANZ, El Renacimiento sacro en la diócesis de Osma-Soria. 1.1, Burgo de Osma, 1979, p. 23.

11. M. D. TEIJEIRA PABlos, Juan de Bruselas y la sillería coral de la Catedral de Zamora, Zamora, 1996.

12. On peut trouver ce nom écrit avec des variantes différentes: Felipe de Borgoña, Bigarny, Vigarny, Vigarni, Viguerny, Biguerny, Bigarne ou Viguernis.

13. P. L. ECHEVERRía GoÑI, «Protagonismo de los maestros galos de la talla en la introducción y evolución del Renacimiento en Navarra », Príncipe de Viana, 73, 256 (2012), p. 515-548.

14. P. BERNARDI, «Entre mobilités sociale et géographique : les pérégrinations d'un tailleur de pierre français dans la Provence du Xve siècle », in Les transferts artistiques..., op. cit., p. 178 : «Dans la Provence urbaine, le "flux revigorant de l'immigration" atteint des proportions notables. À Avignon, près de $71,5 \%$ des personnes qui testent entre 1451 et 1475 sont d'origine étrangère ; elles sont 51,2 \% dans le même temps, à Aix-en-Provence, et $33 \%$ à Arles. La population de constructeurs ne fait, à cet égard, pas exception; la part des immigrés y atteignant à Aix-enProvence, par exemple, 42,5\% des bâtisseurs actifs en 1451-1475. La ville d'Arles - dont Hélion l'Auvergnat est dit citoyen et habitant - voit arriver un grand nombre d'hommes parmi lesquels, à partir de 1450, beaucoup sont originaires du Massif central. »

15. P. JULIEN, "La sculpture toulousaine de la Renaissance: des ateliers itinérants au foyer rayonnant ", in La sculpture française du XvI ${ }^{e}$ siècle..., op. cit., p. 62-79.

16. A. TURCAT, Étienne Jamet, alias Esteban Jamete: sculpteur français de la Renaissance en Espagne, condamné par l'Inquisition, Paris, 1994, p. 396.

17. F. J. GALANTE GÓMEZ, El Cristo de La Laguna : un asesinato, una escultura y un grabado, San Cristóbal de La Laguna, 1999.

18. J. A. SALAS AUSÉNS, "La inmigración francesa a Aragón en la Edad Moderna », Estudios del Departamento de Historia Moderna (1985-1986), p. 51-77.

19. J. Á. BARRIO LOZA, Los Beaugrant en el contexto de la escultura manierista vasca, Bilbao, 1984, p. 160.

20. J. PÉREZ, «El modelo flamenco en Castilla », in R. A. VERDOK et W. THOMAS (éd.), Encuentros en Flandes. Relaciones e intercambios hispanoflamencos a inicios de la Edad Moderna, Soria, 2000, p. 103-115 ; F. VILLASEÑOR SEBASTIÁN, Iconografía marginal en Castilla. 1454-1492, Madrid, 2009.

21. J. M. DE AZCÁRATE RISTORI, Escultura del siglo XVI, Madrid, 1958, p. 395.

22. R. PÉREZ DE CASTRO, «El escultor Pedro de Bolduque : orígenes y primeras obras ", Boletín del Seminario de Estudios de Arte, 78 (2012), p. 71.

23. I. FUENTES REBOlLo, "Vigarny, Picardo y el retablo de la colegiata de Valpuesta (Burgos)", Boletín del Museo Nacional de Escultura, 8 (2004), p. 7-14. 
24. M. J. TARIFA CASTILLA, « Nuevas noticias documentales sobre el entallador renacentista francés Pierres Picart », Artigrama, 27 (2012), p. 395-423.

25. P. NAVASCUÉS PALACIO, «Rodrigo Gil y los entalladores de la fachada de la Universidad de Alcalá », Archivo Español de Arte, 45, 178 (1972), p. 103-118.

26. A. ORICHETA GARCíA, La sillería coral del convento de San Marcos de León, León, 1997, p. 293.

27. A. J. MORALES, «El Ayuntamiento de Sevilla : maestros canteros, entalladores e imagineros », Laboratorio de arte, 4 (1991), p. 61-68.

28. J. A. SALAS AUSÉNS, «Leyes de inmigración y flujos migratorios en la España Moderna », in Los extranjeros en la España Moderna, 2, p. 682 et 687-688.

29. M.J. REDONDO CANTERA, «L'apport français à la sculpture de la Renaissance en Castille. Réflexions sur le style et les matériaux ", in La sculpture française du XVI eiècle..., op. cit., p. 138-149.

30. J. GÓMEZ MARTÍNEZ, « El Renacimiento a la francesa en la obra de los Corral de Villalpando », in R. PÉREZ DE CASTRO y M. GARCÍA MARBÁn (coord.), Cultura y arte en Tierra de Campos. I Jornadas de Medina de Rioseco en su historia, Valladolid, 2001, p. 131-151.

31. J. M. TORRES PÉREZ, « Un claro seguidor de Roque Balduque : Pedro de Paz, escultor extremeño del siglo XVI », in VI Congreso de estudios extremeños, Cáceres, 1981, p. 301-309.

32. H. ZERNER, L'art de la Renaissance en France : l'invention du classicisme, Paris, 1996, p. 414.

33. S. MUNOZ, «Les têtes en médaillon dans le décor sculpté de la Renaissance: typologie et diffusion d'un ornement à travers la France méridionale et l'Espagne », in J. LUGAND, Les échanges artistiques..., op. cit., p. 165-182.

34. R. LÓPEZ TORRIJOS, «Las medallas y la visión del mundo clásico en el siglo XVI español », in La visión del mundo clásico en el arte español. Actas de las VI Jornadas de Arte, Madrid, 1993, p. 93-104.

35. A. CASTRO SANTAMARÍA, "Algunas aportaciones sobre la Catedral de Plasencia », Norba-Arte, 14-15 (1994-1995), p. 287-296.

36. J. GÓMEZ MARTíNEZ, « El Renacimiento a la francesa en la obra de los Corral de J. GÓMEZ MARTínEZ, «El Renacimiento a la francesa en la obra de los Corral de Villalpando », in Cultura y arte en Tierra de Campos, p.131-151; J. IBÁÑEZ FERNÁNDEZ, "Renaissance à la française dans le quinientos aragonais", in J. LUGAND, Les échanges artistiques..., op. cit., p. 55-81; M. J. REDONDO CANTERA, « L'apport français à la sculpture... », op. cit., p. 143.

\section{RÉSUMÉS}

L'arrivée d'un grand nombre de sculpteurs en Espagne tout au long du XVI ${ }^{\mathrm{e}}$ siècle est bien connue. Pour la majorité d'entre eux, il s'agit de Français qui déménagent afin de se développer professionnellement et qui s'installent surtout en Castille. Même si cette tendance démarre au Moyen Âge, ces tailleurs sont beaucoup plus nombreux pendant le second tiers du XVI ${ }^{\mathrm{e}}$ siècle. Ils ne se limitent pas seulement à introduire des ornements italianisants dans la sculpture de l'époque : ces maîtres établissent des ateliers, où ils montrent leurs techniques et savoirs aux jeunes apprentis.

Renaissance sculpture in Spain welcomes an important migration flow of carvers from northern countries, mainly from France; most of them settle down in the former Kingdom of Castile. Despite this trend starting in the Middle Ages, it is along the $16^{\text {th }}$ century when this stream is more numerous. Not only have they played an important role in the introduction of Italian-style 
ornamental items, but also opened their own workshops, where new apprentices were taught. Many of these foreign carvers enjoyed a solid professional reputation and developed a highly successful career.

INDEX

Mots-clés : sculpture, mobilité, XVe-XVIe siècles, atelier, huchiers

Keywords : sculpture, mobility, 15th-16th centuries, workshop, huchiers

\section{AUTEUR}

MARÍA TERESA RODRÍGUEZ BOTE

Université de Salamanque 